\title{
Phase Structural Analysis of Ultra High-Molecular Weight Polyethylene Fibers by Solid State High Resolution NMR
}

\author{
Atsushi KaJi, Yasuo Ohta, Hiroshi Yasuda, and Masao Murano \\ Katata Research Center, Toyobo Co., Ltd., \\ 2-1-1 Katata Otsu, Shiga, 520-02, Japan
}

(Received August 7, 1989)

\begin{abstract}
C}$ solid state high resolution NMR spectra of ultra high-molecular weight polyethylene fibers (UHF) were measured. The main narrow line is due to orthorhombic crystalline phase (ORC). Because of the high degree of crystallinity, amorphous phase signal is observed so small as the foot of the main crystalline phase signal. The small signal of monoclinic crystal phase (MCC) can be observed at lower field than the ORC signal. Signal width of MCC peak is as narrow as ORC peak. Several kinds of relaxation parameters are measured to discuss the two crystalline and intermediate phase. The crystalline phase signal can be divided into several components according to the difference in relaxation time. The signal of highly mobile component spreads over MCC and ORC signals. This component is not a crystal but its conformation is similar to that of the crystal. It may be such as oriented amorphous.
\end{abstract}

KEY WORDS Ultra High-Molecular Weight Polyethylene / Solid-State NMR / CP-MAS / Phase Structure / $T_{1}$ / Orthorhombic Crystal / Monoclinic Crystal /

There are various kinds of high performance fibers. It is known that those fibers can be produced from rigid chain polymers such as aramid or PBT (polyphenylenbenzobisthiazole). ${ }^{1}$ Chain extension and alignment along the fiber direction are important for getting high modulus and high strength and are comparatively easy for these polymers. Ultra high molecular weight polyethylene (UHMWPE, $M_{w} \geqq 10^{6}$ ) is one of flexible polymers but shows extremely high modulus and high strength. On drawing a single crystal mat of UHMWPE, the modulus increases to $220 \mathrm{GPa}$ which is close to the theoretical value of perfect crystal. $^{2}$ It means that the molecules assume the fully extended structure. UHMWPE can be drawn also from solution or gel, giving high modulus ( $\geqq 100 \mathrm{GPa})$ and high strength ( $\geqq 3.0 \mathrm{GPa}$ ) fibers. ${ }^{3-5}$ This process is interesting from a practical point of view though mechanical properties of the fibers obtained by this method are a little inferior to those obtained by drawing single crystal mat.

Melt drawing of UHMWPE is very difficult because of high melt viscosity and high performance fibers could not be obtained by this method. ${ }^{6}$

The mechanical properties of UHMWPE fibers owe to their high crystallinity and large crystal size. So, it is important to investigate the structure both in the crystalline and amorphous phases.

Linear polyethylene (high density polyethylene, HDPE) including UHMWPE is one of the crystalline polymers, and its crystal structure has been studied by X-ray diffraction and other methods. ${ }^{7}$ The orthorhombic form (ORC) is obtained in ambient conditions and transforms to the monoclinic form (MCC) under special conditions. ${ }^{7,8}$ The molecular conformation in these two crystalline phases is all-trans planar zigzag. The two phases differ from each other in the lateral chain arrangement. The molecular planes are nearly 
perpendicular in ORC, while they are parallel to one another in MCC.

By combining such various techniques as high power proton decoupling (dipole decoupling, DD), magic angle spinning (MAS), and cross polarization (CP), solid state high resolution ${ }^{13} \mathrm{C}$ NMR spectra can be measured. Then, solid state high resolution NMR is one of the powerful methods to elucidate the structure in the crystalline and amorphous phases of polymers, and has been applied to various samples of polyethylene. In the case of polyethylene, there are observed a narrow signal due to ORC at $33 \mathrm{ppm}$ and a broad signal due to amorphous phase (NC) at about $31.2 \mathrm{ppm}$. The latter value is close to the isotropic chemical shift. ${ }^{9}$ The chemical shift and the width of the NC signal are due to the existence of the gauche forms distributed along the polymer chain in a random way. The conformation of a particular bond is changed with time between the trans and the gauche forms, but the rate of the exchange is not so high as to cause the narrowing of the NMR signal. The MCC signal is observed at about $34.3 \mathrm{ppm} .{ }^{10-12}$ The difference in the chemical shift between two crystalline forms has been explained by a theoretical calculation. ${ }^{13}$ There are many kinds of NMR relaxation parameters corresponding to the various types of molecular mobility. ${ }^{14}$

$T_{1}$ of carbon $\left(T_{1 \mathrm{c}}\right)$ in crystalline phase is extremely longer than that of NC. The $T_{1 \mathrm{C}}$ decay curve is not reproducible by a single exponential function but consists of three components having different mobilities. ${ }^{11}$

UHMWPE is being studied by various methods to elucidate its excellent properties. The studies of solid state high resolution NMR applied to UHMWPE, especially that in the state of high performance fibers, are rather limited. ${ }^{10}$

In this work, we applied this technique to UHMWPE fibers and investigated the structure of both the crystalline and amorphous phases. Our efforts were concentrated to get information about "the oriented amorphous phase" which is assumed to exist in highly oriented polymer materials.

\section{EXPERIMENTAL}

\section{Materials}

UHMWPE $\left(M_{w} \fallingdotseq 2 \times 10^{6}\right)$ fibers (UHF) were produced by gel drawing according to Smith et $a l .{ }^{15}$ The crystallinity of UHF estimated from density was about $90 \%$. The tensile modulus was about $130 \mathrm{GPa}$ and the strength was about $3 \mathrm{GPa}$. Cast film of UHMWPE (UOF) was also prepared from solution. The crystallinity of UOF estimated from density was about $60 \%$. HDPE $\left(M_{w} \fallingdotseq\right.$ $6 \times 10^{4}$ ) fiber (HDF) was prepared by melt drawing through the method of Capaccio et al. ${ }^{16}$ The crystallinity of HDF estimated from density was about $65 \%$. The modulus and strength of HDF were about $40 \mathrm{GPa}$ and $1 \mathrm{GPa}$, respectively.

\section{NMR Measurement}

Solid state high resolution ${ }^{13} \mathrm{C}$ NMR spectra were measured using a Varian XL-300 $\left({ }^{13} \mathrm{C}\right.$, $75.5 \mathrm{MHz}$ ) at ambient temperature. Dipole decoupling power was about $50 \mathrm{kHz}$. Careful adjustment of spectrometer was necessary to get sufficiently narrow line width (about $30 \mathrm{~Hz}$ for ORC signal in UHF). Magic angle sample spinning rate was about $3.5 \mathrm{kHz}$.

${ }^{13} \mathrm{C}$ NMR signals were divided into several Lorentzians by the least squares method. We measured the following relaxation parameters; $T_{1}$ of proton $\left(T_{1 \mathrm{H}}\right)$ from carbon signal through CP-MAS and $T_{1}$ of carbon $\left(T_{1 \mathrm{C}}\right)$ by Torchia's method. ${ }^{17}$

\section{RESULTS AND DISCUSSION}

\section{${ }^{13}$ C NMR Spectra}

Typical CP-MAS spectra of UHF, UOF, and HDF are shown in Figure 1. The main peak is due to ORC. The NC signal is observed at higher field. In UHF, the NC signal is too small to be detectable. The tail of the ORC peak 


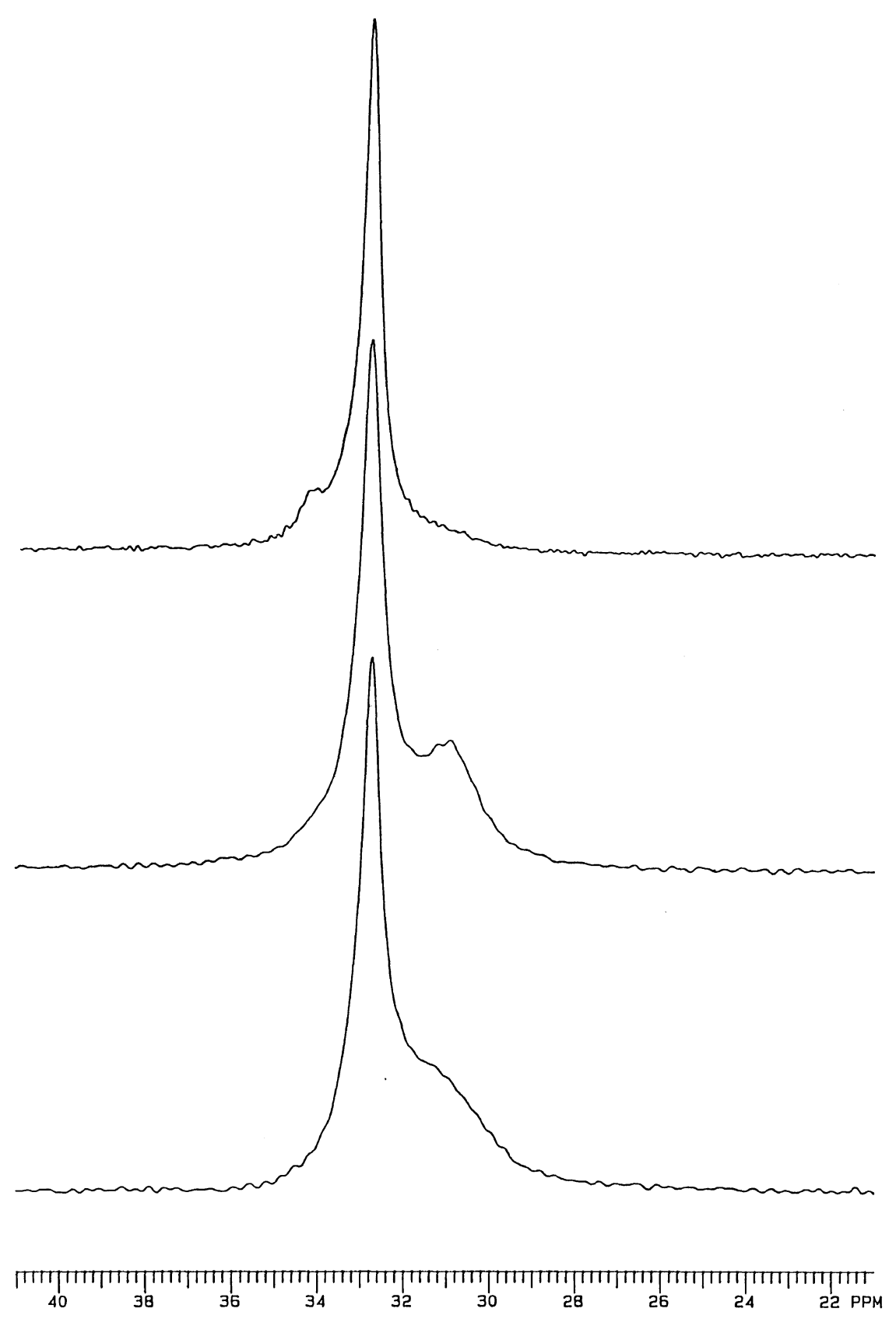

Figure 1. ${ }^{13} \mathrm{C}$ CP-MAS spectra of UHMWPE: top, UHF; center, UOF; bottom, HDF.

spreads toward higher field where the NC signal should be found. The MCC signal can also be seen at lower field side of the ORC signal. The ORC signal in UHF has a half width of about $30 \mathrm{~Hz}(0.4 \mathrm{ppm})$ which is narrower than that in other samples. The broadening of the ORC signal in UOF or HDF suggests that there might exist some dispersity in the crystalline phase. These spectra were separated into three or four Lorentzians by the least squares method 

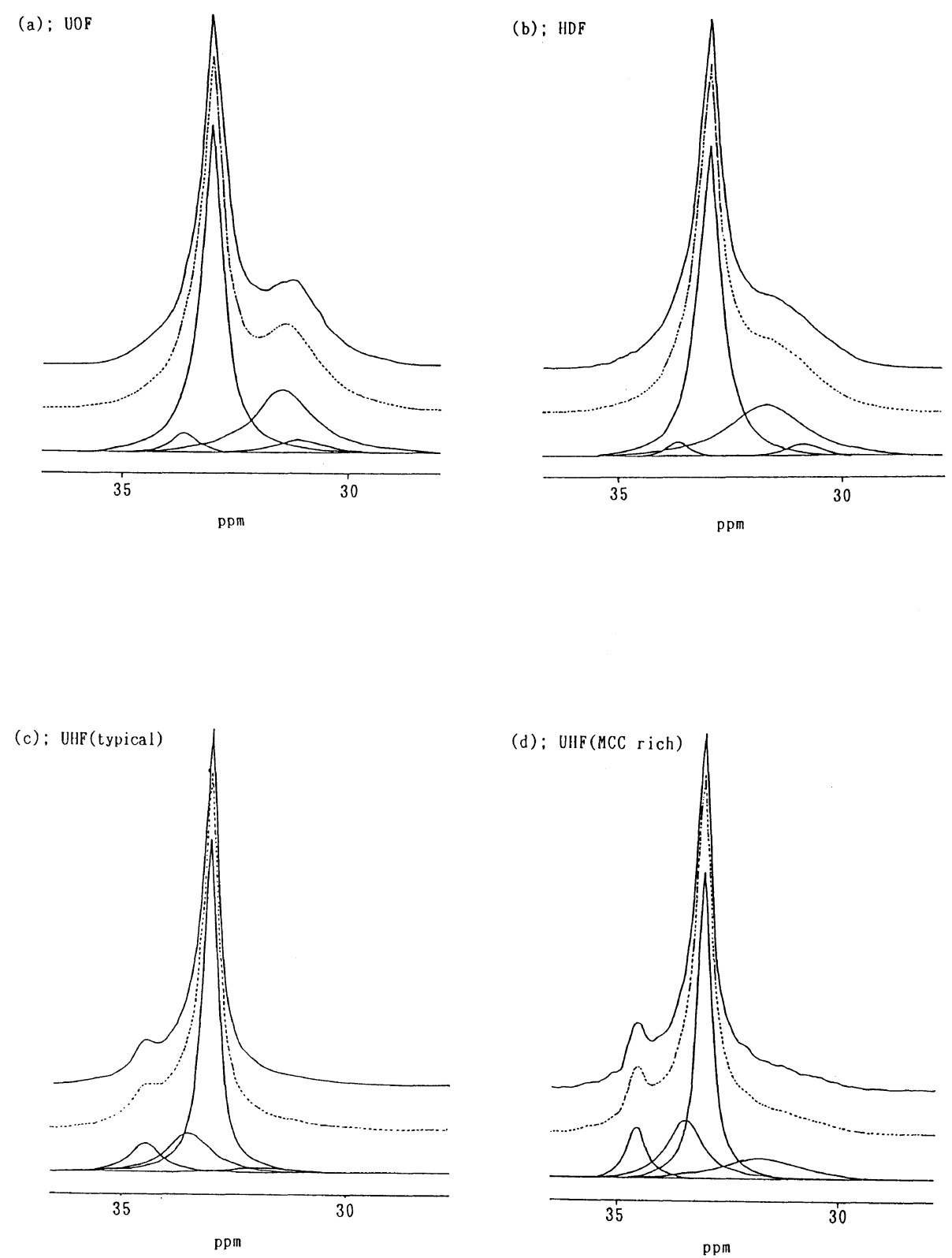

Figure 2. Separation of ${ }^{13} \mathrm{C}$ CP-MAS spectra by some Lorentzians: top, original spectra; center, sum of Lorentzians; bottom, stacked plot of Lorentzians. (a), UOF; (b), HDF; (c), UHF (normal); UHF (MCC rich).

as shown in Figure 2. Kitamaru et al. divided the spectra of variously crystallized polyethylene samples into four components such as two crystalline (ORC and MCC), the rubberlike amorphous and the interfacial phases by profile analysis. Each signal of ORC and MCC was assumed to be a single Lorentzian. The interfacial phase signal centered at $31.3 \mathrm{ppm}$ can be distinguished from the rubber-like amorphous phase signal centered at $31.0 \mathrm{ppm}$. 
Table I. Relaxation times of PE samples

\begin{tabular}{|c|c|c|c|c|c|c|c|c|c|}
\hline \multirow{3}{*}{ UHF } & \multicolumn{3}{|c|}{$T_{1 \mathrm{H}} / \mathrm{s}$} & \multicolumn{3}{|c|}{$T_{1 \rho \mathrm{c}} / \mathrm{ms}$} & \multicolumn{3}{|c|}{$T_{1 \mathrm{c}} / \mathrm{s}$} \\
\hline & $\mathrm{MCC}$ & ORC & $\mathrm{NC}$ & MCC & ORC & NC & $\mathrm{MCC} / \%$ & ORC/\% & $\mathrm{NC}$ \\
\hline & 4.5 & 4.9 & 4.0 & 0.30 & 0.49 & 4.0 & $\begin{array}{r}2060(82) \\
257(18)\end{array}$ & $\begin{array}{rr}2090 & (85) \\
115 \quad(11) \\
4.1 & (4)\end{array}$ & 0.22 \\
\hline UOF & & 1.3 & 0.9 & & 0.55 & 4.2 & & $\begin{array}{r}1480 \quad(77) \\
66.3(23)\end{array}$ & 0.33 \\
\hline $\mathrm{HDF}$ & & 1.4 & 1.0 & & 0.58 & 5.2 & & $\begin{array}{rr}472 & (50) \\
43.7(20) \\
1.4(15)\end{array}$ & 0.48 \\
\hline
\end{tabular}

The difference in the NC signal between UOF and HDF is regarded due to differences in the ratios of the two components. In the case of UHF, the rubber-like amorphous could not be observed. In this respect, the UHF is similar to the solution-grown crystal in the literature. ${ }^{8}$ The shape of MCC signal in UHF can be seen by signal separation as shown in Figure 2(c). The signal width of MCC is almost the same as that of ORC. It is confirmed by the spectrum of a MCC-rich sample (Figure 2(d)). It is found that a better fitting could be obtained when the fourth component was inserted between the MCC and ORC peaks and this fourth peak was broader than the crystal peaks. This component should be included in the crystalline region because the crystallinity of UHF is about $90 \%$ and the area of the NC signal alone occupies a percentage enough for the amorphous region.

\section{Relaxation Time Measurement}

Various kinds of relaxation times $\left(T_{1}\right.$ of proton $\left(T_{1 \mathrm{H}}\right), T_{1 \rho}$ of carbon $\left(T_{1 \rho \mathrm{C}}\right)$ and $T_{1}$ of carbon $\left(T_{1 C}\right)$ ) were measured to make clear the structure of the crystalline and intermediate phases in relation to molecular mobility, and they are listed in Table I.

$T_{1 \mathrm{H}}$ is strongly influenced by proton spin diffusion. $T_{1 \mathrm{H}}$ of the crystalline phase is almost the same as that of the amorphous phase. $T_{1 \mathrm{H}}$ values of UHF are longer than those of HDF and UOF. $T_{1 \mathrm{H}}$ of $\mathrm{MCC}$ is the same as that of ORC in UHF. Crystallinity of UHF is so high that the amorphous phase becomes extremely small. Then, the $T_{1 \mathrm{H}}$ value of the amorphous phase approaches that of the crystalline phase. In HDF or UOF, the crystallite size is so small that the $T_{1 \mathrm{H}}$ is shortened through spin diffusion to the amorphous phase.

$T_{1 \rho \mathrm{C}}$ of the crystalline phase is shorter than that of the amorphous phase by the contribution of $T_{2}$ relaxation caused by insufficient spin lock power (about $24 \mathrm{kHz}$ ). The difference in $T_{1 \rho \mathrm{C}}$ between the crystalline and amorphous phases can be used to separate the spectra to partial spectra of the crystalline and amorphous phases. MCC has the same $T_{1 \rho \mathrm{C}}$ as ORC.

$T_{1 \mathrm{C}}$ changed from a few thousand seconds to less than 0.5 second depending on molecular mobility and crystallite size. ${ }^{14} \mathrm{~A}$ typical $T_{1 \mathrm{C}}$ decay plot of the crystalline component is shown in Figure 3. The initial part of the decay is nonexponential. This effect is due to a distribution of relaxation times. The decay is exponential at delay time greater than a few hundred seconds. This might be due to spin diffusion of carbon. We divided the decay curve into some components according to the difference in $T_{1 \mathrm{C}}$. These components correspond to different domains present in the sample. The signal due to the crystalline component is composed of the signals due to these domains. 


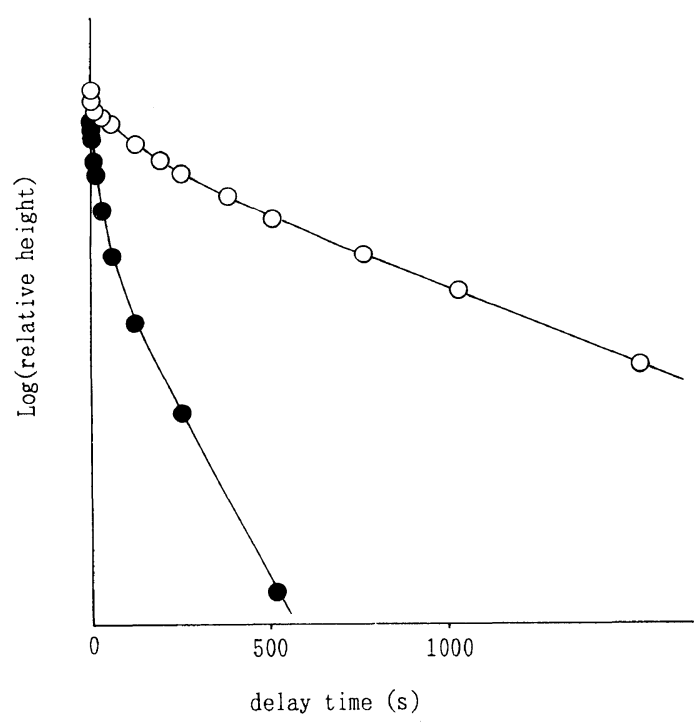

Figure 3. $T_{1 \mathrm{C}}$ decay plot of crystalline component of UHF and HDF: $O$, UHF; 9 , HDF.

In the case of UHF, we considered three domains, named $\mathrm{CC}, \mathrm{BC}$, and $\mathrm{AC}$ in the order of decreasing $T_{1 \mathrm{C}}$. The $T_{1 \mathrm{C}}$ value of $\mathrm{CC}$ is up to a few thousand seconds, that of $\mathrm{BC}$ is about a hundred seconds, and that of $\mathrm{AC}$ is a few seconds. The $T_{1 \mathrm{C}}$ value of $\mathrm{MCC}$ is almost the same as that of ORC.

In the case of HDF, we can also get three components $(\mathrm{CC}, \mathrm{BC}$, and $\mathrm{AC})$ in the same way as the case of UHF. The $T_{1 \mathrm{C}}$ value of CC (longest $T_{1 \mathrm{C}}$ ) in HDF is shorter than in UHF.

The CC component is due to the crystalline phase. The $T_{1 \mathrm{C}}$ value depends on its size. The presence of the mobile phase in the neighborhood of the crystal affects the mobility of the molecules in the crystal, or the spin diffusion effect reduces the $T_{1 \mathrm{c}}$ differences among the domains. $T_{1 \mathrm{C}}$ value of a portion in the crystal changes with the distance from the mboile region.

The defects in the crystal have larger mobility and shorter $T_{1 \mathrm{C}}$ than the other crystalline part. The defects can be regarded those at border of the crystal. The crystal containing more defects can be said to be of smaller size.

The crystal size in HDF is smaller than in
UHF. MCC has similar mobility and size to ORC, or small MCC is surrounded with ORC.

On the other hand, $T_{1 \mathrm{C}}$ of $\mathrm{BC}$ and $\mathrm{AC}$ components depend not only on the crystal size but also on the mobility. BC and AC have smaller size or larger mobility, though the conformation is almost the same as $\mathrm{CC}$ because their chemical shift is about the same as CC.

Separation of Spectra According to the Differences in $T_{1 \mathrm{C}}$

We can draw each spectrum of $\mathrm{CC}, \mathrm{BC}, \mathrm{AC}$, and $\mathrm{NC}$ by the use of different $T_{1 \mathrm{C}}$ values as shown in Figure 4. These spectra are obtained as follows. We obtain first variously relaxed spectra by Torchia's pulse sequence like in the $T_{1 \mathrm{C}}$ measurement with some delay time $\tau$. Then, each spectrum corresponding to the component with individual $T_{1 \mathrm{C}}$ can be derived from linear combination of these partially relaxed spectra. The longest $T_{1 \mathrm{C}}$ component $\mathrm{CC}$ has two narrow lines corresponding to ORC and MCC in UHF. MCC could not be seen in UOF or in HDF. The BC signal has a shoulder at lower field between MCC and ORC peaks. The AC signal is as wide as NC and spreads over MCC and ORC. The chemical shifts of BC and AC signals indicate that these components have similar conformation to CC. Wide signal width comes from the variation of structures. Their shorter $T_{1 \mathrm{C}}$ s mean larger mobility. Some parts of these components should be identical to the additional component when the CP-MAS spectra is separated into a few Lorentzians. They might correspond to the oriented amorphous or intermediate components present between MCC and ORC. In other words, the conformation of the polymer chains are trans zigzag but their lateral packing is not the same as in the crystal.

\section{REFERENCES}

1. S. R. Allen, A. G. Filippov, R. J. Farris, E. L. Thomas, C. P. Wong, G. C. Berry, and E. C. Chenevey, Macromolecules, 14, 1135 (1981). 
Phase Structural Analysis of UHF by Solid State High Resolution NMR
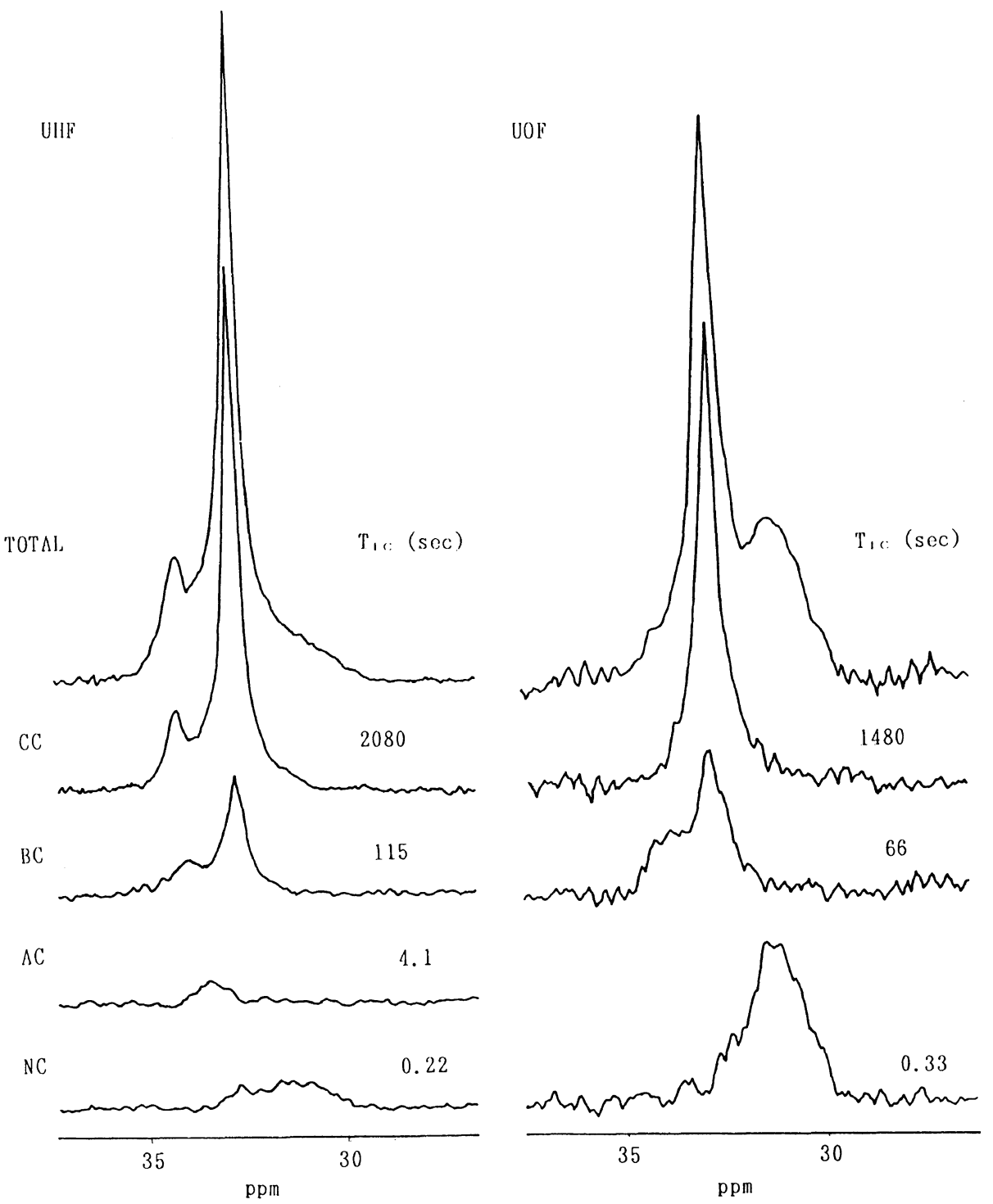

Figure 4. Separation of ${ }^{13} \mathrm{C}$ CP-MAS spectra according to different $T_{1 \mathrm{C}}$ : CC, longest $T_{1 \mathrm{C}}$ component (longer than $\left.1 \times 10^{3} \mathrm{~s}\right)$; BC, middle $T_{1 \mathrm{C}}$ component $\left(\mathrm{ca} .1 \times 10^{2} \mathrm{~s}\right.$ ); AC, shortest $T_{1 \mathrm{C}}$ component in crystalline peak $(1-10 \mathrm{~s})$; NC, non crystalline (amorphous) phase peak (shorter than $1 \mathrm{~s}$ ).

2. T. Kanamoto, A. Tsuruta, K. Tanaka, M. Takeda, and R. S. Porter, Polym. J., 15, 327 (1983).

3. J. Smook, J. C. Torfs, P. F. van Hutten, and A. J. Pennings, Polym. Bull., 2, 293 (1980).

4. P. Smith and P. J. Lemstra, J. Mater. Sci., 15, 505 (1980).

5. J. Smook and A. J. Pennings, J. Mater. Sci., 19, 31 and 3443 (1984).
6. P. J. Lemstra, R. Kirschbaum, T. Ohta, and H. Yasuda, "Developments in Oriented Polymers," Vol. 2, I. M. Ward, Ed., Elsevier Applied Science, London, Chapter 2.

7. T. Seto, T. Hara, and K. Tanaka, Jpn. J. Appl. Phys., 7, 31 (1968).

8. I. L. Hay and A. Keller, J. Polym. Sci., C, 30, 289 (1970). 
9. W. L. Earl and D. L. VanderHart, Macromolecules, 12, 762 (1979).

10. D. L. VanderHart and F. Khoury, Polymer, 25, 1589 (1984).

11. R. Kitamaru, F. Horii, and K. Murayama, Macromolecules, 19, 636 (1986).

12. M. Takenaka, T. Yamanobe, T. Komoto, I. Ando, and H. Sato, Solid State Commun., 61, 563 (1987).

13. T. Yamanobe, T. Sorita, T. Komoto, I. Ando, and H. Sato, J. Mol. Structure, 131, 267 (1985).

14. S. Ganapathy, V. P. Chacko, and R. G. Bryant,
Macromolecules, 19, 1021 (1986).

15. P. Smith and P. J. Lemstra; US Patent, 4,344,908 (1982).

16. G. Capaccio, F. Smith, and I. M. Ward, Japan Patent, 50-126921 (1975).

17. D. A. Torchia, J. Magn. Reson., 30, 613 (1978).

18. D. L. VanderHart and A. N. Garroway, J. Chem. Phys., 71, 2773 (1979).

19. D. E. Axelson, L. Mandelkern, R. Popli, and P. Matieu, J. Polym. Sci., Polym. Phys. Ed., 21, 2319 (1983). 